\title{
Individual ergodic theorem for intuitionistic fuzzy observables using IF-probability
}

\author{
Katarína Čunderlíková \\ Mathematical Institute, Slovak Academy of Sciences \\ Štefánikova 49, 81473 Bratislava, Slovakia \\ e-mail: cunderlikova.lendelova@gmail.com
}

Received: 22 September 2019

Accepted: 13 October 2019

\begin{abstract}
The aim of this paper is to formulate the individual ergodic theorem for intuitionistic fuzzy observables using $\mathcal{P}$-almost everywhere convergence, where $\mathcal{P}$ is an intuitionistic fuzzy probability. Since the intuitionistic fuzzy probability can be decomposed to two intuitionistic fuzzy states, we can use the results holding for intuitionistic fuzzy states.
\end{abstract}

Keywords: Intuitionistic fuzzy event, Intuitionistic fuzzy observable, Intuitionistic fuzzy probability, Product, $\mathcal{P}$-almost everywhere convergence, $\mathcal{P}$-preserving transformation, Individual ergodic theorem.

2010 Mathematics Subject Classification: 03B52, 60A86, 60B10, 28 D05.

\section{Introduction}

In [1, 2], K. T. Atanassov introduced the notion of intuitionistic fuzzy sets. Later P. Grzegorzewski and E. Mrówka defined the probability on the family of intuitionistic fuzzy events

$$
\mathcal{N}=\left\{\left(\mu_{A}, \nu_{A}\right) ; \mu_{A}, \nu_{A} \text { are } \mathcal{S} \text {-measurable and } \mu_{A}+\nu_{A} \leq 1_{\Omega}\right\}
$$

as a mapping $\mathcal{P}$ from the family $\mathcal{N}$ to the set of all compact intervals in $R$ by the formula

$$
\mathcal{P}\left(\left(\mu_{A}, \nu_{A}\right)\right)=\left[\int_{\Omega} \mu_{A} d P, 1-\int_{\Omega} \nu_{A} d P\right],
$$

where $(\Omega, \mathcal{S}, P)$ is the probability space, see [7]. This intuitionistic fuzzy probability was axiomatically characterized by B. Riečan (see [10]). 
In this paper, we formulate the Individual ergodic theorem for intuitionistic fuzzy observables, using $\mathcal{P}$-almost everywhere convergence, where $\mathcal{P}$ is an intuitionistic fuzzy probability. Recall that the formulation of the individual ergodic theorem for intuitionistic fuzzy events with product first appeared in the paper [3]. There we used a separating intuitionistic fuzzy probability. Since the intuitionistic fuzzy probability $\mathcal{P}$ can be decomposed to two intuitionistic fuzzy states, we can use the results holding for intuitionistic fuzzy states, which were proved in [6].

Remark that in a whole text we use a notation IF as an abbreviation for intuitionistic fuzzy.

\section{IF-events, IF-states, IF-observables and IF-mean value}

In this section we explain the basic notions from IF-probability theory, see $[1,2,13,14,15]$.

Definition 2.1. Let $\Omega$ be a nonempty set. An IF-set $\mathbf{A}$ on $\Omega$ is a pair $\left(\mu_{A}, \nu_{A}\right)$ of mappings $\mu_{A}, \nu_{A}: \Omega \rightarrow[0,1]$ such that $\mu_{A}+\nu_{A} \leq 1_{\Omega}$.

Definition 2.2. Start with a measurable space $(\Omega, \mathcal{S})$. Hence $\mathcal{S}$ is a $\sigma$-algebra of subsets of $\Omega$. An IF-event is called an IF-set $\mathbf{A}=\left(\mu_{A}, \nu_{A}\right)$ such that $\mu_{A}, \nu_{A}: \Omega \rightarrow[0,1]$ are $\mathcal{S}$-measurable.

The family of all IF-events on $(\Omega, \mathcal{S})$ will be denoted by $\mathcal{F}, \mu_{A}: \Omega \longrightarrow[0,1]$ will be called the membership function, $\nu_{A}: \Omega \longrightarrow[0,1]$ will be called the non-membership function.

If $\mathbf{A}=\left(\mu_{A}, \nu_{A}\right) \in \mathcal{F}, \mathbf{B}=\left(\mu_{B}, \nu_{B}\right) \in \mathcal{F}$, then we define the Łukasiewicz binary operations $\oplus, \odot$ on $\mathcal{F}$ by

$$
\begin{aligned}
& \left.\mathbf{A} \oplus \mathbf{B}=\left(\left(\mu_{A}+\mu_{B}\right) \wedge 1_{\Omega},\left(\nu_{A}+\nu_{B}-1\right) \vee 0_{\Omega}\right)\right) \\
& \left.\mathbf{A} \odot \mathbf{B}=\left(\left(\mu_{A}+\mu_{B}-1\right) \vee 0_{\Omega},\left(\nu_{A}+\nu_{B}\right) \wedge 1_{\Omega}\right)\right)
\end{aligned}
$$

and the partial ordering is given by

$$
\mathbf{A} \leq \mathbf{B} \Longleftrightarrow \mu_{A} \leq \mu_{B}, \nu_{A} \geq \nu_{B}
$$

In the paper, we use max-min connectives defined by

$$
\mathbf{A} \vee \mathbf{B}=\left(\mu_{A} \vee \mu_{B}, \nu_{A} \wedge \nu_{B}\right),
$$

$$
\mathbf{A} \wedge \mathbf{B}=\left(\mu_{A} \wedge \mu_{B}, \nu_{A} \vee \nu_{B}\right)
$$

and the de Morgan rules

$$
\begin{aligned}
& (a \vee b)^{*}=a^{*} \wedge b^{*}, \\
& (a \wedge b)^{*}=a^{*} \vee b^{*},
\end{aligned}
$$

where $a^{*}=1-a$.

Example 2.3. A fuzzy set $f: \Omega \longrightarrow[0,1]$ can be regarded as an IF-set if we put

$$
\mathbf{A}=\left(f, 1_{\Omega}-f\right)
$$


If $f=\chi_{A}$, then the corresponding IF-set has the form

$$
\mathbf{A}=\left(\chi_{A}, 1_{\Omega}-\chi_{A}\right)=\left(\chi_{A}, \chi_{A^{\prime}}\right) .
$$

In this case $\mathbf{A} \oplus \mathbf{B}$ corresponds to the union of sets, $\mathbf{A} \odot \mathbf{B}$ to the intersection of sets and $\leq$ to the set inclusion.

Consider a probability space $(\Omega, \mathcal{S}, P)$. Then in [7] the IF-probability $\mathcal{P}(\mathbf{A})$ of an IF-event $\mathbf{A}=\left(\mu_{A}, \nu_{A}\right) \in \mathcal{F}$ has been defined as a compact interval by the equality

$$
\mathcal{P}(\mathbf{A})=\left[\int_{\Omega} \mu_{A} d P, 1-\int_{\Omega} \nu_{A} d P\right] .
$$

Let $\mathcal{J}$ be the family of all compact intervals. Then the mapping $\mathcal{P}: \mathcal{F} \rightarrow \mathcal{J}$ can be defined axiomatically similarly as in [10].

Definition 2.4. Let $\mathcal{F}$ be the family of all IF-events in $\Omega$. A mapping $\mathcal{P}: \mathcal{F} \rightarrow \mathcal{J}$ is called an IF-probability if the following conditions hold:

(i) $\mathcal{P}\left(\left(1_{\Omega}, 0_{\Omega}\right)\right)=[1,1], \mathcal{P}\left(\left(0_{\Omega}, 1_{\Omega}\right)\right)=[0,0]$;

(ii) If $\mathbf{A} \odot \mathbf{B}=\left(0_{\Omega}, 1_{\Omega}\right)$, then $\mathcal{P}(\mathbf{A} \oplus \mathbf{B})=\mathcal{P}(\mathbf{A})+\mathcal{P}(\mathbf{B})$;

(iii) If $\mathbf{A}_{n} \nearrow \mathbf{A}$, then $\mathcal{P}\left(\mathbf{A}_{n}\right) \nearrow \mathcal{P}(\mathbf{A})$.

(Recall that $\left[\alpha_{n}, \beta_{n}\right] \nearrow[\alpha, \beta]$ means that $\alpha_{n} \nearrow \alpha, \beta_{n} \nearrow \beta$, but $\mathbf{A}_{n}=\left(\mu_{A_{n}}, \nu_{A_{n}}\right) \nearrow \mathbf{A}=$ $\left(\mu_{A}, \nu_{A}\right)$ means $\mu_{A_{n}} \nearrow \mu_{A}, \nu_{A_{n}} \searrow \nu_{A}$.)

IF-probability $\mathcal{P}$ is called separating, if

$$
\mathcal{P}\left(\left(\mu_{A}, \nu_{A}\right)\right)=\left[\mathcal{P}^{b}\left(\mu_{A}\right), 1-\mathcal{P}^{\sharp}\left(\nu_{A}\right)\right],
$$

where the functions $\mathcal{P}^{b}, \mathcal{P}^{\sharp}: \mathcal{T} \rightarrow[0,1]$ are probabilities.

Of course, each $\mathcal{P}(\mathbf{A})$ is an interval, denote it by $\mathcal{P}(\mathbf{A})=\left[\mathcal{P}^{b}(\mathbf{A}), \mathcal{P}^{\sharp}(\mathbf{A})\right]$. By this way we obtain two functions

$$
\mathcal{P}^{b}: \mathcal{F} \rightarrow[0,1], \mathcal{P}^{\sharp}: \mathcal{F} \rightarrow[0,1]
$$

and some properties of $\mathcal{P}$ can be characterized by some properties of $\mathcal{P}^{b}, \mathcal{P}^{\sharp}$, see [11].

Theorem 2.5. Let $\mathcal{P}: \mathcal{F} \rightarrow \mathcal{J}$ and $\mathcal{P}(\mathbf{A})=\left[\mathcal{P}^{b}(\mathbf{A}), \mathcal{P}^{\sharp}(\mathbf{A})\right]$ for each $\mathbf{A} \in \mathcal{F}$. Then $\mathcal{P}$ is an IF-probability if and only if $\mathcal{P}^{b}$ and $\mathcal{P}^{\sharp}$ are IF-states.

Proof. See [11, Theorem 2.3]

Recall that by an intuitionistic fuzzy state (IF-state) $\mathbf{m}$ we understand each mapping $\mathbf{m}$ : $\mathcal{F} \rightarrow[0,1]$ which satisfies the following conditions (see [12]):

(i) $\mathbf{m}\left(\left(1_{\Omega}, 0_{\Omega}\right)\right)=1, \mathbf{m}\left(\left(0_{\Omega}, 1_{\Omega}\right)\right)=0$;

(ii) if $\mathbf{A} \odot \mathbf{B}=\left(0_{\Omega}, 1_{\Omega}\right)$ and $\mathbf{A}, \mathbf{B} \in \mathcal{F}$, then $\mathbf{m}(\mathbf{A} \oplus \mathbf{B})=\mathbf{m}(\mathbf{A})+\mathbf{m}(\mathbf{B})$;

(iii) if $\mathbf{A}_{n} \nearrow \mathbf{A}$ (i.e. $\left.\mu_{A_{n}} \nearrow \mu_{A}, \nu_{A_{n}} \searrow \nu_{A}\right)$, then $\mathbf{m}\left(\mathbf{A}_{n}\right) \nearrow \mathbf{m}(\mathbf{A})$. 
Now we introduce the notion of an observable. Let $\mathcal{J}$ be the family of all intervals in $R$ of the form

$$
[a, b)=\{x \in R: a \leq x<b\} .
$$

Then the $\sigma$-algebra $\sigma(\mathcal{J})$ is denoted by $\mathcal{B}(R)$ and it is called the $\sigma$-algebra of Borel sets, its elements are called Borel sets (see [16]).

Definition 2.6. By an IF-observable on $\mathcal{F}$ we understand each mapping $x: \mathcal{B}(R) \rightarrow \mathcal{F}$ satisfying the following conditions:

(i) $x(R)=\left(1_{\Omega}, 0_{\Omega}\right), x(\emptyset)=\left(0_{\Omega}, 1_{\Omega}\right)$;

(ii) If $A \cap B=\emptyset$ and $A, B \in \mathcal{B}(R)$, then $x(A) \odot x(B)=\left(0_{\Omega}, 1_{\Omega}\right)$ and $x(A \cup B)=x(A) \oplus x(B)$;

(iii) If $A_{n} \nearrow A$ and $A_{n}, A \in \mathcal{B}(R), n \in N$, then $x\left(A_{n}\right) \nearrow x(A)$.

Similarly, we can define the notion of $n$-dimensional IF-observable.

Definition 2.7. By an n-dimensional IF-observable on $\mathcal{F}$ we understand each mapping $x: \mathcal{B}\left(R^{n}\right) \rightarrow \mathcal{F}$ satisfying the following conditions:

(i) $x\left(R^{n}\right)=\left(1_{\Omega}, 0_{\Omega}\right), x(\emptyset)=\left(0_{\Omega}, 1_{\Omega}\right)$;

(ii) If $A \cap B=\emptyset$ and $A, B \in \mathcal{B}\left(R^{n}\right)$, then $x(A) \odot x(B)=\left(0_{\Omega}, 1_{\Omega}\right)$ and $x(A \cup B)=$ $x(A) \oplus x(B) ;$

(iii) If $A_{n} \nearrow A$ and $A_{n}, A \in \mathcal{B}\left(R^{n}\right), n \in N$, then $x\left(A_{n}\right) \nearrow x(A)$.

Similarly, as in the classical case the following theorem can be proved $([9,15])$.

Theorem 2.8. Let $x: \mathcal{B}(R) \longrightarrow \mathcal{F}$ be an IF-observable, $\mathbf{m}: \mathcal{F} \longrightarrow[0,1]$ be an IF-state. Define the mapping $\mathbf{m}_{x}: \mathcal{B}(R) \longrightarrow[0,1]$ by the formula

$$
\mathbf{m}_{x}(C)=\mathbf{m}(x(C))
$$

Then $\mathbf{m}_{x}: \mathcal{B}(R) \longrightarrow[0,1]$ is a probability measure.

Since $\mathbf{m}_{x}: \mathcal{B}(R) \rightarrow[0,1]$ plays now an analogous role as $P_{\xi}: \mathcal{B}(R) \rightarrow[0,1]$, we can define IF-expected value $\mathbf{E}(x)$ by the same formula (see [9]).

Definition 2.9. We say that an IF-observable $x$ is an integrable IF-observable if the integral $\int_{R} t d \mathbf{m}_{x}(t)$ exists. In this case, we define the IF-expected value

$$
\mathbf{E}(x)=\int_{R} t d \mathbf{m}_{x}(t) .
$$

If the integral $\int_{R} t^{2} d \mathbf{m}_{x}(t)$ exists, then we define IF-dispersion $\mathbf{D}^{2}(x)$ by the formula

$$
\mathbf{D}^{2}(x)=\int_{R} t^{2} d \mathbf{m}_{x}(t)-(\mathbf{E}(x))^{2}=\int_{R}(t-\mathbf{E}(x))^{2} d \mathbf{m}_{x}(t) .
$$




\section{Product operation, joint IF-observable and function of several IF-observables}

In [8] we introduced the notion of product operation on the family of IF-events $\mathcal{F}$, and showed an example of this operation.

Definition 3.1. We say that a binary operation $\cdot$ on $\mathcal{F}$ is product if it satisfies the following conditions:

(i) $\left(1_{\Omega}, 0_{\Omega}\right) \cdot\left(a_{1}, a_{2}\right)=\left(a_{1}, a_{2}\right)$ for each $\left(a_{1}, a_{2}\right) \in \mathcal{F}$;

(ii) The operation - is commutative and associative;

(iii) If $\left(a_{1}, a_{2}\right) \odot\left(b_{1}, b_{2}\right)=\left(0_{\Omega}, 1_{\Omega}\right)$ and $\left(a_{1}, a_{2}\right),\left(b_{1}, b_{2}\right) \in \mathcal{F}$, then

$$
\left(c_{1}, c_{2}\right) \cdot\left(\left(a_{1}, a_{2}\right) \oplus\left(b_{1}, b_{2}\right)\right)=\left(\left(c_{1}, c_{2}\right) \cdot\left(a_{1}, a_{2}\right)\right) \oplus\left(\left(c_{1}, c_{2}\right) \cdot\left(b_{1}, b_{2}\right)\right)
$$

and

$$
\left(\left(c_{1}, c_{2}\right) \cdot\left(a_{1}, a_{2}\right)\right) \odot\left(\left(c_{1}, c_{2}\right) \cdot\left(b_{1}, b_{2}\right)\right)=\left(0_{\Omega}, 1_{\Omega}\right)
$$

for each $\left(c_{1}, c_{2}\right) \in \mathcal{F}$;

(iv) If $\left(a_{1 n}, a_{2 n}\right) \searrow\left(0_{\Omega}, 1_{\Omega}\right),\left(b_{1 n}, b_{2 n}\right) \searrow\left(0_{\Omega}, 1_{\Omega}\right)$ and $\left(a_{1 n}, a_{2 n}\right),\left(b_{1 n}, b_{2 n}\right) \in \mathcal{F}$, then $\left(a_{1 n}, a_{2 n}\right) \cdot\left(b_{1 n}, b_{2 n}\right) \searrow\left(0_{\Omega}, 1_{\Omega}\right)$.

The following theorem defines the product operation for IF-events.

Theorem 3.2. The operation · defined by

$$
\left(x_{1}, y_{1}\right) \cdot\left(x_{2}, y_{2}\right)=\left(x_{1} \cdot x_{2}, y_{1}+y_{2}-y_{1} \cdot y_{2}\right)
$$

for each $\left(x_{1}, y_{1}\right),\left(x_{2}, y_{2}\right) \in \mathcal{F}$ is product operation on $\mathcal{F}$.

Proof. See [8, Theorem 1].

In [13] B. Riečan defined the notion of a joint IF-observable and he proved its existence.

Definition 3.3. Let $x, y: \mathcal{B}(R) \rightarrow \mathcal{F}$ be two IF-observables. The joint IF-observable of the IF-observables $x$, y is a mapping $h: \mathcal{B}\left(R^{2}\right) \rightarrow \mathcal{F}$ satisfying the following conditions:

(i) $h\left(R^{2}\right)=\left(1_{\Omega}, 0_{\Omega}\right), h(\emptyset)=\left(0_{\Omega}, 1_{\Omega}\right)$;

(ii) If $A, B \in \mathcal{B}\left(R^{2}\right)$ and $A \cap B=\emptyset$, then $h(A \cup B)=h(A) \oplus h(B)$ and $h(A) \odot h(B)=\left(0_{\Omega}, 1_{\Omega}\right)$;

(iii) If $A, A_{1}, \ldots \in \mathcal{B}\left(R^{2}\right)$ and $A_{n} \nearrow A$, then $h\left(A_{n}\right) \nearrow h(A)$;

(iv) $h(C \times D)=x(C) \cdot y(D)$ for each $C, D \in \mathcal{B}(R)$. 
Theorem 3.4. For each two IF-observables $x, y: \mathcal{B}(R) \rightarrow \mathcal{F}$ there exists their joint IF-observable. Proof. See [13, Theorem 3.3].

Remark 3.5. The joint IF-observable of the IF-observables $x$, y from Definition 3.3 is a twodimensional IF-observable.

If we have several IF-observables and a Borel measurable function, we can define the IF-observable, which is the function of several IF-observables. About this says the following definition.

Definition 3.6. Let $x_{1}, \ldots, x_{n}: \mathcal{B}(R) \rightarrow \mathcal{F}$ be IF-observables, $h_{n}$ be their joint IF-observable and $g_{n}: R^{n} \rightarrow R$ be a Borel measurable function. Then, we define the IF-observable $g_{n}\left(x_{1}, \ldots, x_{n}\right): \mathcal{B}(R) \rightarrow \mathcal{F}$ by the formula

$$
g_{n}\left(x_{1}, \ldots, x_{n}\right)(A)=h_{n}\left(g_{n}^{-1}(A)\right) .
$$

for each $A \in \mathcal{B}(R)$.

\section{Lower and upper limits, $\mathcal{P}$-almost everywhere convergence}

In [4] we defined the notions of lower and upper limits for a sequence of IF-observables.

Definition 4.1. We shall say that a sequence $\left(x_{n}\right)_{n}$ of IF-observables has $\limsup _{n \rightarrow \infty}$ if there exists an IF-observable $\bar{x}: \mathcal{B}(R) \rightarrow \mathcal{F}$ such that

$$
\bar{x}((-\infty, t))=\bigvee_{p=1}^{\infty} \bigvee_{k=1}^{\infty} \bigwedge_{n=k}^{\infty} x_{n}\left(\left(-\infty, t-\frac{1}{p}\right)\right)
$$

for every $t \in R$. We write $\bar{x}=\limsup x_{n}$.

Note that if another IF-observable y satisfies the above condition, then $\mathbf{m} \circ y=\mathbf{m} \circ \bar{x}$.

Definition 4.2. A sequence $\left(x_{n}\right)_{n}$ of IF-observables has $\liminf _{n \rightarrow \infty}$ if there exists an IF-observable $\underline{x}$ such that

$$
\underline{x}((-\infty, t))=\bigvee_{p=1}^{\infty} \bigwedge_{k=1}^{\infty} \bigvee_{n=k}^{\infty} x_{n}\left(\left(-\infty, t-\frac{1}{p}\right)\right)
$$

for all $t \in R$. Notation: $\underline{x}=\liminf _{n \rightarrow \infty} x_{n}$.

In paper [5] we showed the connection between two kinds of $\mathcal{P}$-almost everywhere convergence.

Definition 4.3. Let $\left(x_{n}\right)_{n}$ be a sequence of IF-observables on an $I F$-space $(\mathcal{F}, \mathcal{P})$. We say that $\left(x_{n}\right)_{n}$ converges $\mathcal{P}$-almost everywhere to 0 , if

$$
\begin{aligned}
\mathcal{P}\left(\bigwedge_{p=1}^{\infty} \bigvee_{k=1}^{\infty} \bigwedge_{n=k}^{\infty} x_{n}\left(\left(-\frac{1}{p}, \frac{1}{p}\right)\right)\right) & =\lim _{p \rightarrow \infty} \lim _{k \rightarrow \infty} \lim _{i \rightarrow \infty} \mathcal{P}\left(\bigwedge_{n=k}^{k+i} x_{n}\left(\left(-\frac{1}{p}, \frac{1}{p}\right)\right)\right)= \\
& =[1,1]=1 .
\end{aligned}
$$


Remark 4.4. The defining formula is equivalent to the following equality

$$
\mathcal{P}\left(\bigvee_{p=1}^{\infty} \bigwedge_{k=1}^{\infty} \bigvee_{n=k}^{\infty} x_{n}\left(R \backslash\left(-\frac{1}{p}, \frac{1}{p}\right)\right)\right)=[0,0]=0
$$

Theorem 4.5. A sequence $\left(x_{n}\right)_{n}$ of IF-observables converges $\mathcal{P}$-almost everywhere to 0 if and only if it converges $\mathcal{P}^{b}$-almost everywhere and $\mathcal{P}^{\sharp}$-almost everywhere to 0.

Proof. See [5, Theorem 5].

Proposition 4.1. A sequence $\left(x_{n}\right)_{n}$ of IF-observables converges $\mathcal{P}$-almost everywhere to 0 if and only if

$$
\begin{aligned}
\mathcal{P}\left(\bigvee_{p=1}^{\infty} \bigvee_{k=1}^{\infty} \bigwedge_{n=k}^{\infty} x_{n}\left(\left(-\infty, t-\frac{1}{p}\right)\right)\right) & =\mathcal{P}\left(\bigvee_{p=1}^{\infty} \bigwedge_{k=1}^{\infty} \bigvee_{n=k}^{\infty} x_{n}\left(\left(-\infty, t-\frac{1}{p}\right)\right)\right)= \\
& =\mathcal{P}\left(0_{\mathcal{F}}((-\infty, t))\right)
\end{aligned}
$$

for every $t \in R$.

Proof. See [5, Proposition 2].

In accordance to Proposition 4.1, we can extend the notion of $\mathcal{P}$-almost everywhere convergence in the following way.

Definition 4.6. A sequence $\left(x_{n}\right)_{n}$ of IF-observables converges $\mathcal{P}$-almost everywhere to an IFobservable $x$, if

$$
\begin{aligned}
\mathcal{P}\left(\bigvee_{p=1}^{\infty} \bigvee_{k=1}^{\infty} \bigwedge_{n=k}^{\infty} x_{n}\left(\left(-\infty, t-\frac{1}{p}\right)\right)\right) & =\mathcal{P}\left(\bigvee_{p=1}^{\infty} \bigwedge_{k=1}^{\infty} \bigvee_{n=k}^{\infty} x_{n}\left(\left(-\infty, t-\frac{1}{p}\right)\right)\right)= \\
& =\mathcal{P}(x((-\infty, t)))
\end{aligned}
$$

for every $t \in R$.

Sometimes we need to work with a sequence of IF-observables induced by a Borel measurable function.

Recall, that the corresponding probability spaces are $\left(R^{N}, \sigma(\mathcal{C}), P^{b}\right)$ and $\left(R^{N}, \sigma(\mathcal{C}), P^{\sharp}\right)$, where $\mathcal{C}$ is the family of all sets of the form

$$
\left\{\left(t_{i}\right)_{i=1}^{\infty}: t_{1} \in A_{1}, \ldots, t_{n} \in A_{n}\right\}
$$

and $P^{b}, P^{\sharp}$ are the probability measures determined by the equalities

$$
\begin{aligned}
& P^{b}\left(\left\{\left(t_{i}\right)_{i=1}^{\infty}: t_{1} \in A_{1}, \ldots, t_{n} \in A_{n}\right\}\right)=\mathcal{P}^{b}\left(x_{1}\left(A_{1}\right) \cdot \ldots \cdot x_{n}\left(A_{n}\right)\right), \\
& P^{\sharp}\left(\left\{\left(t_{i}\right)_{i=1}^{\infty}: t_{1} \in A_{1}, \ldots, t_{n} \in A_{n}\right\}\right)=\mathcal{P}^{\sharp}\left(x_{1}\left(A_{1}\right) \cdot \ldots \cdot x_{n}\left(A_{n}\right)\right) .
\end{aligned}
$$

The corresponding projections $\xi_{n}: R^{N} \rightarrow R$ are defined by the equality

$$
\xi_{n}\left(\left(t_{i}\right)_{i=1}^{\infty}\right)=t_{n} .
$$


Theorem 4.7. Let $\left(x_{n}\right)_{n}$ be a sequence of IF-observables, $\left(\xi_{n}\right)_{n}$ be the sequence of corresponding projections, $\left(g_{n}\right)_{n}$ be a sequence of Borel measurable functions $g_{n}: R^{n} \rightarrow R$. If the sequence $\left(g_{n}\left(\xi_{1}, \ldots, \xi_{n}\right)\right)_{n}$ converges $P^{b}$-almost everywhere and $P^{\sharp}$-almost everywhere, then the sequence $\left(g_{n}\left(x_{1}, \ldots, x_{n}\right)\right)_{n}$ converges $\mathcal{P}$-almost everywhere and

$$
\mathcal{P}\left(\limsup _{n \rightarrow \infty} g_{n}\left(x_{1}, \ldots, x_{n}\right)((-\infty, t))\right)=\mathcal{P}\left(\liminf _{n \rightarrow \infty} g_{n}\left(x_{1}, \ldots, x_{n}\right)((-\infty, t))\right)
$$

for each $t \in R$. Moreover

$$
\mathcal{P}\left(\limsup _{n \rightarrow \infty} g_{n}\left(x_{1}, \ldots, x_{n}\right)((-\infty, t))\right)=\left[P^{b}(E), P^{\sharp}(E)\right]
$$

for each $t \in R$, where $E=\left\{u \in R^{N}: \lim _{\sup _{n \rightarrow \infty}} g_{n}\left(\xi_{1}(u), \ldots, \xi_{n}(u)\right)<t\right\}$.

Proof. See [5, Theorem 6].

\section{Individual Ergodic Theorem}

In paper [5] we proved the modification of the classical Individual Ergodic Theorem using malmost everywhere convergence. Since the intuitionistic fuzzy probability $\mathcal{P}$ can be decomposed to two intuitionistic fuzzy states $\mathbf{m}$ (see $[11,14]$ ), then we try to formulate the modification of the classical Individual Ergodic Theorem using $\mathcal{P}$-almost everywhere convergence.

Now, we recall the modification of the Individual Ergodic Theorem for the IF-state (see [6]).

Theorem 5.1. (Individual Ergodic Theorem) Let $(\mathcal{F}, \cdot)$ be a family of IF-events with product, and $\mathbf{m}$ be an IF-state. Let $x$ be an integrable IF-observable and $\tau$ be an $\mathbf{m}$-preserving transformation. Then there exists an integrable IF-observable $x^{*}$ such that

(i) $\mathbf{E}(x)=\mathbf{E}\left(x^{*}\right)$,

(ii) $\lim _{n \rightarrow \infty} \frac{1}{n} \sum_{i=0}^{n-1}\left(\tau^{i} \circ x\right)=x^{*} \mathbf{m}$-almost everywhere.

Proof. See [6, Theorem 6.3] .

We defined the IF-mean value of an IF-observable and $\mathcal{P}$-almost everywhere convergence in the previous sections. Now we must define a transformation preserving an intuitionistic probability $\mathcal{P}$.

Definition 5.2. Let $(\mathcal{F}, \cdot)$ be a family of $I F$-events with product, $\mathcal{P}$ be an IF-probability. Then, a mapping $\tau: \mathcal{F} \rightarrow \mathcal{F}$ is said to be a $\mathcal{P}$-preserving transformation if the following conditions are satisfied:

(i) $\tau\left(\left(1_{\Omega}, 0_{\Omega}\right)\right)=\left(1_{\Omega}, 0_{\Omega}\right)$;

(ii) If $\mathbf{A}, \mathbf{B} \in \mathcal{F}$ and $\mathbf{A} \odot \mathbf{B}=\left(0_{\Omega}, 1_{\Omega}\right)$, then $\tau(\mathbf{A}) \odot \tau(\mathbf{B})=\left(0_{\Omega}, 1_{\Omega}\right)$ and $\tau(\mathbf{A} \oplus \mathbf{B})=\tau(\mathbf{A}) \oplus \tau(\mathbf{B}) ;$ 
(iii) If $\mathbf{A}_{n} \nearrow \mathbf{A}, \mathbf{A}_{n}, \mathbf{A} \in \mathcal{F}, n \in N$, then $\tau\left(\mathbf{A}_{n}\right) \nearrow \tau(\mathbf{A})$;

(iv) $\mathcal{P}(\tau(\mathbf{A}) \cdot \tau(\mathbf{B}))=\mathcal{P}(\mathbf{A} \cdot \mathbf{B})$ for each $\mathbf{A}, \mathbf{B} \in \mathcal{F}$.

Now we show the connection to the $\mathbf{m}$-preserving transformation. Recall that by $\mathbf{m}$-preserving transformation we understand each mapping $\tau: \mathcal{F} \rightarrow \mathcal{F}$ if the following conditions are satisfied:

(i) $\tau\left(\left(1_{\Omega}, 0_{\Omega}\right)\right)=\left(1_{\Omega}, 0_{\Omega}\right)$;

(ii) If $\mathbf{A}, \mathbf{B} \in \mathcal{F}$ and $\mathbf{A} \odot \mathbf{B}=\left(0_{\Omega}, 1_{\Omega}\right)$, then $\tau(\mathbf{A}) \odot \tau(\mathbf{B})=\left(0_{\Omega}, 1_{\Omega}\right)$ and $\tau(\mathbf{A} \oplus \mathbf{B})=\tau(\mathbf{A}) \oplus \tau(\mathbf{B}) ;$

(iii) If $\mathbf{A}_{n} \nearrow \mathbf{A}, \mathbf{A}_{n}, \mathbf{A} \in \mathcal{F}, n \in N$, then $\tau\left(\mathbf{A}_{n}\right) \nearrow \tau(\mathbf{A})$;

(iv) $\mathbf{m}(\tau(\mathbf{A}) \cdot \tau(\mathbf{B}))=\mathbf{m}(\mathbf{A} \cdot \mathbf{B})$ for each $\mathbf{A}, \mathbf{B} \in \mathcal{F}$.

See [6].

Theorem 5.3. Let $(\mathcal{F}, \cdot)$ be a family of IF-events with product, $\mathcal{P}$ be an IF-probability. The mapping $\tau: \mathcal{F} \rightarrow \mathcal{F}$ is the $\mathcal{P}$-preserving transformation if and only if the mapping $\tau$ is the $\mathcal{P}^{b}$-preserving transformation and the $\mathcal{P}^{\sharp}$-preserving transformation, where $\mathcal{P}^{b}, \mathcal{P}^{\sharp}$ are the IF-states.

Proof. " $\Rightarrow$ " Let $\mathcal{P}$ be an IF-probability. Then by Theorem 2.5 it can be decomposed to two IF-states $\mathcal{P}^{b}, \mathcal{P}^{\sharp}$ such that $\mathcal{P}(\mathbf{A})=\left[\mathcal{P}^{b}(\mathbf{A}), \mathcal{P}^{\sharp}(\mathbf{A})\right]$ for each $\mathbf{A} \in \mathcal{F}$. If the mapping $\tau: \mathcal{F} \rightarrow \mathcal{F}$ is the $\mathcal{P}$-preserving transformation, then by (iv) from Definition 5.2 we have

$$
\begin{aligned}
{\left[\mathcal{P}^{b}(\mathbf{A} \cdot \mathbf{B}), \mathcal{P}^{\sharp}(\mathbf{A} \cdot \mathbf{B})\right] } & =\mathcal{P}(\mathbf{A} \cdot \mathbf{B})=\mathcal{P}(\tau(\mathbf{A}) \cdot \tau(\mathbf{B})) \\
& =\left[\mathcal{P}^{b}(\tau(\mathbf{A}) \cdot \tau(\mathbf{B})), \mathcal{P}^{\sharp}(\tau(\mathbf{A}) \cdot \tau(\mathbf{B}))\right] .
\end{aligned}
$$

Hence,

$$
\begin{aligned}
& \mathcal{P}^{b}(\tau(\mathbf{A}) \cdot \tau(\mathbf{B}))=\mathcal{P}^{b}(\mathbf{A} \cdot \mathbf{B}), \\
& \mathcal{P}^{\sharp}(\tau(\mathbf{A}) \cdot \tau(\mathbf{B}))=\mathcal{P}^{\sharp}(\mathbf{A} \cdot \mathbf{B}),
\end{aligned}
$$

for each $\mathbf{A}, \mathbf{B} \in \mathcal{F}$. Therefore, $\tau$ is a $\mathcal{P}^{b}$-preserving transformation and a $\mathcal{P}^{\sharp}$-preserving transformation.

“ $\Leftarrow$ " The opposite direction can be proved similarly.

Theorem 5.4. (Individual Ergodic Theorem) Let $(\mathcal{F}, \cdot)$ be a family of IF-events with product, $\mathcal{P}$ be an IF-probability. Let $x$ be an integrable IF-observable and $\tau$ be an $\mathcal{P}$-preserving transformation. Then there exists an integrable IF-observable $x^{*}$ such that

(i) $\mathbf{E}^{b}(x)=\mathbf{E}^{b}\left(x^{*}\right), \mathbf{E}^{\sharp}(x)=\mathbf{E}^{\sharp}\left(x^{*}\right)$

(ii) $\lim _{n \rightarrow \infty} \frac{1}{n} \sum_{i=0}^{n-1}\left(\tau^{i} \circ x\right)=x^{*}, \quad \mathcal{P}$-almost everywhere. 
Proof. Let $\mathcal{P}$ be an IF-probability. By Theorem 2.5 it can be decomposed to two IF-states $\mathcal{P}^{b}, \mathcal{P}^{\sharp}$, such that $\mathcal{P}(\mathbf{A})=\left[\mathcal{P}^{b}(\mathbf{A}), \mathcal{P}^{\sharp}(\mathbf{A})\right]$ for each $\mathbf{A} \in \mathcal{F}$. Let $\tau$ be the $\mathcal{P}$-preserving transformation. Then from Theorem 5.3 we obtain that $\tau$ is the $\mathcal{P}^{b}$-preserving transformation and the $\mathcal{P}^{\sharp}$-preserving transformation, where $\mathcal{P}^{b}, \mathcal{P}^{\sharp}$ are the IF-states. Hence by Theorem 5.1 there exists an integrable IF-observable $x^{*}$ such that

(i) $\mathbf{E}^{b}(x)=\mathbf{E}^{b}\left(x^{*}\right), \mathbf{E}^{\sharp}(x)=\mathbf{E}^{\sharp}\left(x^{*}\right)$

(ii) $\lim _{n \rightarrow \infty} \frac{1}{n} \sum_{i=0}^{n-1}\left(\tau^{i} \circ x\right)=x^{*}, \quad \mathcal{P}^{b}$-almost everywhere and $\mathcal{P}^{\sharp}$-almost everywhere.

Finally by Theorem 5.3 we obtain that

$$
\lim _{n \rightarrow \infty} \frac{1}{n} \sum_{i=0}^{n-1}\left(\tau^{i} \circ x\right)=x^{*}, \quad \mathcal{P} \text {-almost everywhere. }
$$

\section{Conclusion}

The paper is concerned in ergodic theory for family of intuitionistic fuzzy events. We proved the Individual ergodic theorem for intuitionistic fuzzy observables using $\mathcal{P}$-almost everywhere convergence, where $\mathcal{P}$ is an intuitinistic fuzzy probability. The results are a generalization of results given in [3].

\section{References}

[1] Atannasov, K. (1999). Intuitionistic Fuzzy Sets: Theory and Applications, Springer Physica Verlag, Heidelberg.

[2] Atanassov, K. (2012). On Intuitionistic Fuzzy Sets, Springer, Berlin.

[3] Čunderlíková, K. (2010). The individual ergodic theorem on the IF-events with product, Soft Computing - A Fusion of Foundations, Methodologies and Applications, Springer - Verlag, 24 (3), 229-234.

[4] Čunderlíková, K. (2018). Upper and lower limits and m-almost everywhere convergence of intuitionistic fuzzy observables, Notes on Intuitionistic Fuzzy Sets, 24 (4), 40-49.

[5] Čunderlíková, K. (2019). Intuitionistic fuzzy probability and almost everywhere convergence, Proceedings of IWIFSGN 2019, submitted.

[6] Čunderlíková, K. (2019). Individual ergodic theorem for intuitionistic fuzzy observables using IF-state, Iranian Journal of Fuzzy Sets, submitted.

[7] Grzegorzewski, P., \& Mrówka, E. (2002). Probability of intuistionistic fuzzy events, Soft Metods in Probability, Statistics and Data Analysis, P. Grzegorzewski et al. eds., Physica Verlag, New York, 105-115. 
[8] Lendelová, K. (2006). Conditional IF-probability, Advances in Soft Computing: Soft Methods for Integrated Uncertainty Modelling, 275-283.

[9] Lendelová K., \& Riečan B. (2004). Weak law of large numbers for IF-events, Current Issues in Data and Knowledge Engineering, Bernard De Baets et al. eds., EXIT, Warszawa, 309314.

[10] Riečan, B. (2003). A descriptive definition of the probability on intuitionistic fuzzy sets, EUSFLAT '2003, M. Wagenecht, R. Hampet eds., Zittau-Goerlitz Univ. Appl. Sci., 263266.

[11] Riečan, B. (2005). On the probability on IF-sets and MV-algebras, Notes on intuitionistic fuzzy sets, 11 (6), 21-25.

[12] Riečan, B. (2006). On a problem of Radko Mesiar: general form of IF-probabilities, Fuzzy Sets and Systems, 152, 1485-1490.

[13] Riečan, B. (2006). On the probability and random variables on IF events, Applied Artifical Intelligence, Proc. 7th FLINS Conf. Genova, D. Ruan et al. eds., 138-145.

[14] Riečan, B. (2007). Probability theory on intuitionistic fuzzy events, Algebraic and Prooftheoretic aspects of Non-classical Logics, Papers in honour of Daniele Mundici's 60th birthday, Lecture Notes in Computer Science, Vol. 4460, 290-308.

[15] Riečan, B. (2012). Analysis of fuzzy logic models, Intelligent systems (V. Koleshko ed.), INTECH, 219-244.

[16] Riečan, B., \& Neubrunn, T. (1997). Integral, Measure, and Ordering, Kluwer Academic Publishers, Dordrecht and Ister Science, Bratislava. 